\title{
LES PROJETS INTÉGRATEURS FINAUX EN GÉNIE INFORMATIQUE ET LOGICIEL: UN CONTEXTE D'APPRENTISSAGE FAVORISANT LA TRANSITION VERS LE MARCHÉ DU TRAVAIL
}

\author{
Olivier Gendreau et Jérôme Collin \\ Polytechnique Montréal \\ Département de génie informatique et génie logiciel \\ \{olivier.gendreau, jerome.collin\}@ polymtl.ca
}

\begin{abstract}
Résumé - En 2005, Polytechnique Montréal a introduit dans son cursus l'apprentissage par projet. Les projets intégrateurs finaux en génie informatique et logiciel visent le développement complet d'un système, dont les besoins sont précisés par un client industriel, en respectant les pratiques recommandées des domaines respectifs. Cet article explique comment l'implication d'un client industriel dans un projet intégrateur final contribue au processus d'apprentissage des étudiants et facilite leur transition vers le marché du travail. Contrairement à la grande majorité des travaux effectués dans le cadre de leur formation, les étudiants doivent composer avec des besoins changeants du client. Les projets visent d'ailleurs à consolider les acquis de leur formation et de solliciter leurs aptitudes d'apprentissage dans une perspective "juste à temps », propre au contexte industriel, en plus de mettre en pratique leurs habiletés personnelles et relationnelles.
\end{abstract}

Mots clés: apprentissage par projet, projet intégrateur, génie informatique, génie logiciel.

\section{INTRODUCTION}

En 2005, Polytechnique Montréal a introduit dans son cursus l'apprentissage par projet. Parmi les raisons expliquant ce virage pédagogique se trouvent l'importance du développement des habiletés de travail en équipe, ainsi que le développement de l'autonomie des étudiants. Le corps professoral et la direction, aidés de spécialistes, se sont alors lancés dans un important chantier de révision de l'enseignement de l'ingénierie au baccalauréat. Il en est ressorti que les projets intégrateurs se retrouvent au cœur des nouveaux programmes [10].

L'apprentissage par projet connaît un important engouement de mise en application depuis environ une décennie. Uniquement dans le domaine des sciences appliquées de la Francophonie, l'Université Libre de Bruxelles [2], l'Université de Sherbrooke [3], l'Université catholique de Louvain [4], l'École Nationale des Sciences Appliquées de Marrakech [6] et l'Université de Liège [9] ont adopté cette approche, pour ne nommer que ces institutions. Le changement d'approche pédagogique de Polytechnique Montréal s'inscrit dans cette tendance.

Cet article présente d'abord les projets intégrateurs en génie informatique et génie logiciel à Polytechnique Montréal, soit les projets intégrateurs de $1^{\mathrm{re}}, 2^{\mathrm{e}}$ et $3^{\mathrm{e}}$ année, ainsi que les projets intégrateurs finaux. Par la suite, il sera expliqué de quelle manière le contexte d'apprentissage des projets intégrateurs finaux favorise la transition vers le marché du travail.

\section{PROJETS INTÉGRATEURS}

À chaque année d'enseignement au baccalauréat, un projet intégrateur a été introduit dans le but de permettre aux étudiants de mettre en pratique la théorie enseignée durant d'autres cours du programme. Le plus souvent, ces cours théoriques sont suivis parallèlement au projet intégrateur ou à la session précédente et parfois même dans les années antérieures, ce qui permet la consolidation de connaissances.

\subsection{Projets intégrateurs de $1^{\text {re }}, 2^{\mathrm{e}}$ et $3^{\mathrm{e}}$ année}

En génie informatique et logiciel, le projet intégrateur de première année est basé sur la conception et l'implémentation, par étapes, d'un programme permettant de contrôler un petit robot mobile. Les étudiants commencent par souder et monter mécaniquement le robot qui leur est livré dans une boîte. Dans les semaines qui suivent, par divers exercices de programmation en langage $\mathrm{C} / \mathrm{C}++$, ils se familiarisent avec l'architecture matérielle d'un microcontrôleur. Par le fait même, les notions acquises durant les deux cours de programmation (procédurale et orientée objet) et les deux cours sur les aspects matériels (logique des systèmes numériques et architecture des micro-ordinateurs) sont mises en pratique dans le cadre du projet. Les méthodes de base de 
développement logiciel du code du robot suivent celles vues dans le cours d'ingénierie logicielle, offert en parallèle au projet intégrateur. À la fin du projet, le robot doit suivre un parcours relativement complexe et l'évaluation se fait devant public.

Le projet intégrateur de deuxième année vise le développement d'un logiciel interactif permettant à l'utilisateur, d'une part, d'éditer une scène en y ajoutant, supprimant ou déplaçant divers objets 3D et d'autre part, de jouer à un jeu mettant en scène ces différents objets régis par certaines règles de jeu. Les étudiants disposent des spécifications des requis du système logiciel (SRS), élaborés par l'équipe académique, qu'ils doivent développer à partir d'une librairie graphique standard et une base de code qui leur est fournie. Les notions enseignées durant les cours de conception logicielle, de structures de données et d'algorithmes, d'analyse et conception d'interfaces utilisateur et d'infographie sont mises en pratique dans le cadre du projet.

Les projets intégrateurs de troisième année, qui sont différents pour les programmes de génie logiciel et de génie informatique, offrent davantage d'autonomie aux étudiants et sont plus complexes que les deux premiers projets intégrateurs. En génie logiciel, l'objectif principal du projet est de procéder à une réingénierie du projet intégrateur de deuxième année de manière à ajouter deux nouvelles fonctionnalités: la possibilité de jouer à plusieurs joueurs en réseau, ainsi que d'éditer la zone de jeu à partir d'une tablette mobile. En génie informatique, la conception des systèmes embarqués est mise à l'avantplan au sein du développement d'un système sur carte électronique à FPGA. Ces deux projets mettent en pratique, entre autres, les notions du cours de réseaux informatiques suivi durant la même année.

Parallèlement aux notions d'ingénierie mises en valeur durant ces projets, des notions complémentaires sont aussi introduites. En première année, des spécialistes présentent aux étudiants des concepts de travail en équipe (organisation de réunions efficaces, leadership, mode d'interactions, etc.). En deuxième et troisième année, des notions de gestion de projet (gestion de risques, planification structurelle, opérationnelle et budgétaire, suivi de l'avancement, direction) sont enseignées et mises en pratique dans le cadre des projets intégrateurs. De plus, au cours des 3 premières années du programme, l'étudiant aura également l'occasion d'effectuer un ou deux stages en entreprise d'environ 12 semaines chacun.

Les trois premiers projets sont élaborés par l'équipe académique, soit les responsables des projets et leurs assistants. Les projets sont conçus de manière à assurer une progression dans leur complexité, autant dans les concepts nécessaires à leur réalisation que dans les méthodes de développement et l'autonomie nécessaires pour y parvenir.

\subsection{Projet intégrateur final}

Le projet intégrateur final ( $4^{\mathrm{e}}$ année) a pour objectif de poursuivre cette progression de complexité et d'autonomie nécessaires, tout en tenant compte du fait qu'il s'agit de la dernière étape avant l'entrée sur le marché du travail pour la très grande majorité des étudiants.

D'un point de vue académique, il devient difficile de définir des projets qui portent spécifiquement sur les notions vues dans les cours théoriques enseignés au courant de la même année scolaire. En effet, les étudiants ne suivent pas tous les mêmes cours, compte tenu de leur spécialisation. De plus, des organisations (à but lucratif ou sans but lucratif) ont divers intérêts à soumettre des projets pour des étudiants en fin de baccalauréat. Les projets intégrateurs finaux proviennent donc de diverses organisations et chaque équipe d'étudiants se voit attribué un projet différent. Contrairement aux trois premiers projets intégrateurs, les projets intégrateurs finaux sont tous des projets uniques, que nul n'a déjà réalisé auparavant, ce qui implique une certaine forme d'inconnu pour tous les intervenants impliqués.

Afin de tenir compte des préférences des étudiants pour certains sujets et aussi des orientations spécialisées dans lesquelles certains se retrouvent, certains projets se déroulent avec une entreprise dans un secteur d'activité particulier. De cette façon, les étudiants trouvent une motivation particulière et peuvent réaliser un projet dans leur champ de spécialité avant de terminer leurs études de baccalauréat en ingénierie.

De plus, les responsables des projets tiennent compte, dans une certaine mesure, des compétences des étudiants lors de la formation des équipes et du jumelage avec les projets. Les étudiants plus compétents peuvent donc se retrouver avec des projets plus complexes, alors que les étudiants moins compétents peuvent se concentrer sur des projets à leur portée.

Les projets retenus doivent être réalisables en 14 semaines par une équipe de 4 à 6 étudiants, à raison d'environ 270 heures de travail par étudiant. Le client doit proposer un projet qui n'est pas critique pour l'entreprise, car les étudiants sont dans un contexte académique d'apprentissage et non un contexte industriel, même si la transition s'effectue progressivement.

L'équipe technique du département de génie informatique et génie logiciel de Polytechnique Montréal met à la disposition des étudiants un environnement de développement couvrant les besoins de base nécessaires à la réalisation des projets. Par contre, si des outils et dispositifs spécialisés sont nécessaires, le client est invité à les fournir. Chaque équipe se voit aussi attribuer, pour la durée du projet, un local de cinq postes de travail avec une table de réunion et un tableau blanc. Il peut arriver que ce local soit partagé par plus d'une équipe dans le cas 
où le nombre d'étudiants inscrits est particulièrement élevé. Les étudiants reçoivent donc leur client et le responsable du cours dans ce local.

Les clients doivent toujours soumettre un projet qui implique minimalement de la conception. Par exemple, des projets qui ne seraient que des analyses de données, des études préliminaires ou des évaluations d'impacts seraient exclus. En génie informatique et génie logiciel, la plupart du temps, le travail demandé requiert de la programmation. Il peut cependant arriver qu'un projet demande peu de programmation, mais tout de même beaucoup de conception, par exemple un projet d'architecture de réseaux d'ordinateurs.

Étant donné qu'un des objectifs principaux du projet intégrateur final est de satisfaire les besoins formulés par un client, le projet n'a pas à être soumis par un expert en informatique ou en logiciel. Dans ces cas, le client ne comprend pas toujours les moyens que les étudiants prennent pour parvenir à réaliser le système demandé. Ainsi, le responsable du projet doit être plus vigilant afin de s'assurer que les étudiants progressent dans la bonne direction, que le client conserve des attentes réalistes et que la communication entre les deux parties se fasse de façon à ce que chacun comprenne bien le langage de l'autre.

\section{CONTEXTE D'APPRENTISSAGE}

Les projets intégrateurs finaux en génie informatique et génie logiciel favorisent la transition vers le marché du travail à plusieurs égards dont notamment le contexte de travail, le type de supervision et l'évaluation des projets.

\subsection{Contexte de travail}

Les projets intégrateurs finaux offrent davantage d'autonomie aux étudiants que pour les autres projets intégrateurs, notamment en ce qui a trait à la définition du projet, au choix du processus de développement et au choix des outils.

3.1.1. Définition du projet. Contrairement aux autres projets intégrateurs, où le «client» est académique, un projet intégrateur final possède un client «réel ». Ainsi, plutôt que d'obtenir des spécifications préparées, analysées et vérifiées par l'équipe académique, les étudiants ont la responsabilité de définir les besoins du client, puis de détailler les exigences de produit. Une des particularités d'un client «réel » est qu'il a tendance à «changer d'idée». En fait, il peut lui arriver de préciser ses besoins avec le temps, surtout dans le cadre d'un processus de rétroaction notamment grâce à une maquette d'interface ou à un prototype.
Cette façon de procéder se rapproche d'un contexte du marché de travail «traditionnel » où le projet est souvent défini de manière plus générale d'abord, pour par la suite être davantage détaillé par les différents intervenants impliqués dans le projet.

\subsubsection{Choix du processus de développement.} Contrairement aux projets intégrateurs précédents, pour lesquels le processus de développement est fortement encadré ou même imposé, les projets intégrateurs finaux permettent aux étudiants de définir leur propre processus de développement, à condition de respecter quelques contraintes générales telles que l'échéancier et certains livrables obligatoires.

En génie logiciel, chaque équipe doit remettre un document de spécifications des exigences, un document d'architecture logicielle, un plan de développement logiciel, un plan de tests, un rapport de résultats de tests et évidemment le code source. Le processus de développement logiciel est libre à chaque équipe, qui pourra opter pour une approche davantage traditionnelle ou plutôt agile. Dans une certaine mesure, cette flexibilité peut s'apparenter au marché du travail. Bien sûr, au sein d'organisation fortement normée, comme les entreprises du domaine de l'aérospatial, les processus utilisés sont davantage traditionnels et rigides. Par contre, les petites entreprises auvrant dans le domaine logiciel offrent souvent une relative flexibilité aux développeurs qui y travaillent, en ce qui a trait à plusieurs aspects du processus de développement utilisé.

3.1.3. Choix des outils. Contrairement aux autres projets intégrateurs, où les outils sont majoritairement imposés, dans le cadre des projets intégrateurs finaux, les étudiants font le choix de leurs outils, bien que l'équipe académique recommande certains outils de gestion de projet et de gestion de configuration.

En ce qui a trait à la gestion de projet, un grand nombre d'outils sont disponibles. Suite à une analyse comparative des différents outils disponibles, les responsables des projets intégrateurs recommandent aux étudiants l'utilisation de Redmine [11]. En effet, cet outil est disponible sous licence GPL, présente une interface web conviviale permettant d'enregistrer des tâches, de préciser leurs liens de dépendance (de manière à établir le diagramme de Gantt), de gérer les feuilles de temps de chaque membre de l'équipe, etc. De plus, un wiki est inclus et utilisé par les étudiants pour documenter des procédures, des comptes rendus de réunions ou des rapports de nature très variée (documents trouvés sur Internet, rapports de tests, etc.). Bien que Redmine soit recommandé, les étudiants disposent de l'autonomie nécessaire pour utiliser l'outil de gestion de projet de leur choix, que ce soit Trac, Jira, Trello, Teambox, ou autre. 
Un projet d'informatique ou logiciel générant un (très) grand nombre de fichiers informatiques, les outils facilitant la gestion de configuration prennent toute leur importance. Des outils de gestion de versions abondamment utilisés de nos jours sont SVN [1] et Git [5], autant par les entreprises que par les groupes de développement de logiciels à code source ouvert. Ces outils sont employés dès la première année de nos programmes en génie informatique et génie logiciel et le projet intégrateur final donne l'opportunité aux étudiants de choisir l'outil de leur choix. Leur utilisation vise le partage de copies de code différentes entre les membres d'une équipe, incluant la possibilité de réunir toutes les modifications en une seule copie maître, le système enregistrant toutes les modifications, à quels moments elles ont été effectuées et par qui. On peut donc reconstituer à tout moment les versions précédentes d'un fichier quelconque, ainsi que son historique. En informatique et en logiciel, ces fonctionnalités peuvent s'avérer capitales.

À l'aide de certains outils de gestion de projet comme Redmine, il est possible d'associer des tâches à des modifications de fichiers contrôlés par SVN ou Git. Ce genre de lien entre la gestion de projet et la gestion de configuration est très utile pour assurer un certain contrôle du processus de développement, tel que la traçabilité des exigences, ce pour quoi il est très répandu en industrie.

Finalement, l'intérêt de permettre aux équipes de choisir leurs propres outils, plutôt que de les imposer, est d'offrir une autonomie accrue, d'inciter à une curiosité intellectuelle et de valoriser l'imputabilité face à leurs choix. Cette démarche s'appuie sur la volonté de donner davantage d'autonomie aux étudiants avec l'évolution du degré de difficulté des projets intégrateurs, dans une perspective de transition vers le marché du travail.

\subsection{Type de supervision}

La supervision des projets intégrateurs repose sur un suivi de l'avancement superviseur-étudiants et clientétudiants.

Le responsable des projets intégrateurs assure un suivi hebdomadaire de chaque équipe. Il s'agit d'un suivi personnalisé d'une durée moyenne de 30 minutes par semaine. L'utilisation par les étudiants d'un outil de gestion de projet facilite le suivi du déroulement du projet par le responsable et s'apparente au travail en industrie d'un gestionnaire de projet ou d'un gestionnaire de programme. Souvent, le responsable consulte l'outil tout juste avant la rencontre hebdomadaire avec l'équipe pour être au courant des dernières tâches et problèmes de l'équipe, de façon à pouvoir faire un suivi efficace et pouvoir mieux la guider. Par exemple, le responsable peut donner une formation ou diriger les étudiants vers les personnes-ressources appropriées, dans une perspective «juste à temps », propre au contexte industriel.

Le client, pour sa part, effectue un suivi variant selon son domaine d'expertise. Bien sûr, le client d'un projet intégrateur est habituellement davantage proactif que la plupart des clients que l'on rencontre en milieu de travail. De plus, il n'est pas rare qu'un client serve de personneressource pour l'équipe de développement relativement au domaine d'application du produit à développer dans le cadre du projet intégrateur.

\section{3. Évaluation des projets}

L'évaluation des projets s'effectue à trois niveaux soit par le client, par le superviseur du projet et par les étudiants eux-mêmes.

Étant donné que le projet intégrateur final vise une transition vers le marché du travail, son évaluation tente de se rapprocher le plus possible d'un contexte d'évaluation de produit en industrie. Un processus d'évaluation flexible a été élaboré de manière à tenir compte de la très grande variété des projets intégrateurs. Ainsi, l'opinion du client prend une place importante dans ce processus d'évaluation. Plus précisément, en fin de projet, le client donne au responsable du projet son appréciation du respect des exigences définies en début de projet et de la qualité du produit livré par les étudiants. En contrepartie, le superviseur du projet tiendra compte de la complexité et du déroulement du projet, notamment en termes de difficultés techniques rencontrées.

Deux présentations orales sont obligatoires pour les étudiants, soit à la mi-projet et à la fin du projet. Lors de la présentation de mi-projet, les étudiants doivent présenter au responsable du cours et au client leur suggestion de solution à la problématique du client. Lors de la présentation orale finale, les étudiants doivent dévoiler le produit développé et offrir une rétroaction critique du projet (difficultés rencontrées, limites au produit, leçons apprises). Au sein de ces présentations orales, la période de questions occupe une place importante puisqu'elle laisse place aux échanges informels entre le client, le responsable du projet et les étudiants. En fait, ces présentations orales s'apparentent dans une certaine mesure à des réunions d'ingénierie, qui ont lieu fréquemment dans le milieu industriel.

La communication écrite constitue une autre source importante permettant l'évaluation des projets. En effet, la documentation écrite permet au responsable du projet et au client de juger de la qualité de la démarche et de la réflexion des étudiants. Tout comme pour les présentations orales, deux jalons sont imposés soit à la misession et à la fin de session. Lors de la mi-session, les étudiants abordent surtout la définition de la problématique du client et la proposition d'une ou plusieurs solutions potentielles. En génie logiciel, ceci 
prend la forme d'un document des spécifications des requis du système logiciel (SRS) et d'un document d'architecture logicielle du système à réaliser. En génie informatique, un rapport aborde les mêmes sujets, mais dans un format moins formel. Lors de la remise de fin de session, l'accent est mis sur la description du système développé, ainsi que le détail des tests effectués, afin de s'assurer que le produit final réponde aux besoins du client. Des documents supplémentaires, comme un guide utilisateur, un guide d'installation et un guide du développeur peuvent aussi être produits par les étudiants, lorsque pertinents. La nature et la forme de la documentation produite par les étudiants sont influencées par la nature de leur projet. Chaque équipe doit vivre avec les particularités de son projet, d'où l'autonomie supplémentaire qui leur est octroyée.

Le dernier aspect du processus d'évaluation est l'évaluation des coéquipiers entre eux à l'aide de l'outil iPeer [12], développé par UBC. À la mi-session et à la fin de session, chaque étudiant procède à l'évaluation de ses coéquipiers et doit commenter ses choix, en plus de s'évaluer lui-même, sur la base de 3 critères (esprit d'équipe, communication et coordination, productivité et compétence). Bien que ce type d'évaluation soit plutôt rare en industrie, il est très utile dans le contexte académique, afin d'éviter que des étudiants peu travaillants bénéficient indument de l'effort de leurs coéquipiers [8].

En somme, bien que le processus d'évaluation ne soit pas identique en tout point à la réalité industrielle, il se démarque des processus d'évaluation des trois projets intégrateurs précédents en accordant une grande importance à l'évaluation du client et en donnant davantage d'autonomie aux étudiants dans leur processus de développement.

\section{CONCLUSION}

Le changement d'approche pédagogique de Polytechnique Montréal, en faveur de l'apprentissage par projet, s'inscrit dans une tendance observée dans la dernière décennie. Au cœur de cette nouvelle approche, le projet intégrateur final a pour objectif de conclure la formation académique des étudiants tout en favorisant la transition vers le marché du travail. Cette transition s'observe à plusieurs égards dont notamment le contexte de travail (la définition du projet, le choix du processus de développement et le choix des outils), le type de supervision (superviseur-étudiants et client-étudiants) et l'évaluation des projets (par le client, par le superviseur et par les étudiants eux-mêmes).

De manière à améliorer la qualité de la formation offerte dans le cadre des projets intégrateurs, la prochaine étape sera d'harmoniser l'approche académique actuelle aux 12 qualités du BCAPG [7].

\section{Remerciements}

Nous tenons à remercier le département de génie informatique et génie logiciel de Polytechnique Montréal pour son soutien relativement à la rédaction de cet article.

\section{Références}

[1] Apache, Subversion [En ligne], http://subversion.apache.org/

[2] Marie Blondeau et coll., "Apprentissage par projet : Réalisation d'une éolienne urbaine en matériaux de récupération," Journal sur l'enseignement des sciences et technologies de l'information et des systèmes, vol. 8, no. Hors Série 1, 2009.

[3] Noël Boutin, Daniel Dalle, Gérard Lachiver et Philippe Mabilleau, "L'apprentissage par problèmes et par projets pour le développement des compétences professionnelles de l'ingénieur : l'exemple des programmes de génie électrique et de génie informatique de l'Université de Sherbrooke," Journal sur l'enseignement des sciences et technologies de l'information et des systèmes, vol. 7, no. Hors Série 1, 2008.

[4] B. Dehez, E. Matagne et V. Kluyskens, "Apprentissage par projet en électrotechnique : optimisation d'une génératrice électrique à aimants permanents intégrée au moteur d'un avion," Journal sur l'enseignement des sciences et technologies de l'information et des systèmes, vol. 7, no. Hors Série 1, 2008.

[5] Git [En ligne], http://git-scm.com/

[6] Damien Grenier et coll., "Apprentissage par projet autour d'un robot mobile : partage d'expériences entre l'Europe et le Maroc.," Journal sur l'enseignement des sciences et technologies de l'information et des systèmes, vol. 7, no. Hors Série 1, 2008.

[7] Ingénieurs Canada, Bureau canadien d'agrément des programmes de genie - Normes et procedures d'agrément, 2011, 86 pp. Accessible le 3 mai 2013 à http://www.engineerscanada.ca/files/w_Accreditation_Criter ia_Procedures_2011.pdf

[8] Lucy Johnston et Lynden Miles, "Assessing contributions to group assignments," Assessment \& Evaluation in Higher Education, vol. 29, no. 6, pp. 751-768, 2004.

[9] F.Plumier, P.Bleus et C.Geuzaine, "Collaboration Industrielle et Enseignement par Projet en Electronique de Puissance," Journal sur l'enseignement des sciences et technologies de l'information et des systèmes, vol. 8, no. Hors Série 1, 2009.

[10] Richard Prégent, Huguette Bernard et Anastassis Kozanitis, Enseigner à l'université dans une approcheprogramme. Montréal, QC : Presses internationales Polytechnique, 2009, 330 pp.

[11] Redmine [En ligne], http://www.redmine.org/

[12] University of British Columbia, iPeer [En ligne], http://ipeer.ctlt.ubc.ca/ 